\title{
The Use of a Rectification Vest Improves Trunk Stabili- ty and Alignment During Hippotherapy in a Child with Dandy-Walker Syndrome: A Case-Study
}

\author{
Natalia Cristina Fiocco ${ }^{1 \#}$, Mirian Oliveira Batista ${ }^{1 \#}$, Janaína Simões de Oliveira Carbinatto ${ }^{1}$, Vaniele \\ Fernanda Foresti ${ }^{1}$, Lilian Teresa Bucken Gobbi ${ }^{2}$ and Marcelo Pinto Pereira ${ }^{2 *}$ \\ ${ }^{1}$ Equoterapia - Rio Claro - SP - Brazil \\ ${ }^{2}$ São Paulo State University (UNESP), Institute of Biosciences, Rio Claro
}

*Corresponding author: Marcelo Pinto Pereira, Posture and Locomotion Studies Laboratory - Physical Education Dep. - UNESP, Av. 24A, 1515; Bela Vista - Rio Claro/SP - Brazil

\section{ARTICLE INFO}

Received: 幽 April 05, 2019

Published: 幽 April 17, 2019

Citation: Natalia C F, Mirian O B, Janaína d O C, Marcelo P P et al. The Use of a Rectification Vest Improves Trunk Stability and Alignment During Hippotherapy in a Child with Dandy-Walker Syndrome: A CaseStudy. Biomed J Sci \& Tech Res 17(1)2019. BJSTR. MS.ID.002953.

Keywords: Equine therapy; Muscle activity; Body control; Syndromic malformation; IV Ventricle malformation

\begin{abstract}
Dandy-Walker syndrome (DWS) involves a series of congenital malformations of the central nervous system. The clinical manifestations include hypotonia, microcephaly, myelomeningocele and hydrocephalus. Hippotherapy is a therapeutic approach that improves balance, postural alignment, modulation of muscle tone and body awareness. Hence hippotherapy is pointed as a complementary therapy for patients with DWS. Hipposuit ${ }^{\circledR}$ is a rectification vest developed to regulate muscle tone, improving postural alignment during hippotherapy. The use of Hipposuit ${ }^{\circledR}$ has been shown to benefit practitioners with cerebral palsy; however, the effects of Hipposuit $\AA$ on patients with DWS have not been investigated. Therefore, the aim of this study was to investigate the effects of Hipposuit ${ }^{\circledR}$ on postural alignment, stability and muscle recruitment level in a child with DWS. A 3-year-old girl with a medical diagnosis of DWS participated in this study. She presented as clinical features: hypotonia and a deficit in trunk control. For the hippotherapy session a male, 15 years-old docile horse (undefined breed) was used. To assess the effects of Hipposuit $($, three conditions were tested: without vest (NoVest), vest without any elastic tension (Vest - used to assess the effects of tactile stimulation) and vest with elastic tension (Vest+Tension). To assess postural alignment six reflexive markers, positioned bilaterally on participant's body, were recorded by camcorders. To assess postural stability, a triaxial accelerometer was positioned on participant's body. Additionally, electromyographic data was obtained from external oblique muscle to assess muscle activation level. The results indicated an increase of $8.3 \%$ in the trunk extension angle in Vest+Tension compared to NoVest $(\mathrm{p}=0.007)$. Additionally, the Vest+Tension condition improved trunk stability (reduction of trunk acceleration), especially in vertical direction $(p=0.025)$. No differences were observed for muscle activation level $(p>0.09)$. Finally, no differences were also observed for all outcomes in Vest. HippusSuitß showed to be effective to improve trunk alignment and stability in a child with DWS during a hippotherapy session, without any 'accommodation' effects of trunk muscles. These findings cannot be attribute to tactile stimulation, since no effects were observed in the Vest condition.
\end{abstract}

\section{Introduction}

Dandy-Walker syndrome (DWS) was fully described in 1921 by WE Dandy [1]. DWS involves a series of congenital malformations of the central nervous system that includes cystic dilation of the fourth ventricle, agenesis or hypoplasia of the cerebellar vermis and supratentorial hydrocephalus [2,3]. This syndrome is more frequent in females with an estimated incidence of 1:25.000 and usually is first observed during childhood [4]. The symptoms vary among patients according to malformation severity, to the location of lesions and according to the size of cystic dilation [2]. However, clinical signs include the increase in the cephalic perimeter and 
the presence of cerebellar signs, such as ataxia or nystagmus, as well as seizures and spasticity or hypotonia [5]. The treatment of DWS is focused on controlling its manifestations and associated comorbidities [2] and might include pharmacological management and surgery [6]. However, complementary therapies to improve patients' quality of life are recommended.

Among these complementary therapies, hippotherapy is an interesting approach. Hippotherapy uses the movements of a horse to provide motor and sensory stimulation [7] and its effectiveness has been confirmed in several mental and physical disabilities, such as cerebral palsy [8,9], autistic behavioral disorders [10], muscular dystrophy [11], among others. Furthermore, hippotherapy improves motor functions as balance, strength, muscle tone control and body awareness $[8,9,12,13]$. Therefore, hippotherapy is pointed as an interesting intervention to DWS patients, since a delay in psychomotor development is a common feature of this disease [2]. Hippotherapy differs from other therapies since it promotes a center of mass displacement in all three-dimensions, stimulating the integration of vestibular, visual and proprioceptive sensorymotor inputs [14]. A proper body position (postural alignment) is crucial to induce an optimal reception of all these stimuli by the patient [14].

This might be a restrain to patients without proper trunk control, as those with DWS. To overcome this limitation and to improve the effects of hippotherapy, an orthosis named HippusSuit $₫$ (a dynamic, rectification vest) was developed with the main objective to improve postural alignment, postural control and general proprioception [14]. This vest is composed of synthetic rubber with polyamide, consisting of integrated vest and shorts, foot positioner, $\mathrm{X}$ and linear strips and safety belt [14]. HippusSuit@ might be used to stimulate or inhibit several muscles groups according to the way, elastic strips are fasten [14]. Among its benefits, HippusSuit $₫$ might improve body-awareness, sensory stimulation, inhibit neurological patterns and improve postural stability during riding [14]. For example, an improvement in body posture and body awareness were noticed in patients with cerebral palsy [14]. However, in this last study [14], a reduction of trunk extenders activation was observed, suggesting an 'accommodation'/disuse effect, which would not be positive to participants with higher levels of hypotonia. Therefore, although the benefits of HippusSuit $\AA$ had been observed in other disabilities [14], its effects on DWS has never been investigated. Therefore, the aim of this single-case study was to investigate the effects of HippusSuit ${ }^{\circledR}$ on postural alignment, trunk stability and muscle activation during a hippotherapy session in a 3-years old girl with a clinical diagnosis of DWS.

\section{Materials and Methods \\ Case Description}

A 3-years old girl (M.P.) participated in this study. M.P. presented moderate hypotonia with predominance in lower limbs, severe trunk control deficit, mainly at lower levels. M.P. also presented low visual acuity, precarious balance during sitting and an inability to sustain posture for a longer period. Finally, M.P. presented closed myelomeningocele at L4 to S3. After explaining all procedures and answering all M.P.'s parents' questions, an informed consent was signed. All procedures were approved by the Ethical Board and followed the Declaration of Helsinki.

\section{Experimental Procedures}

All procedures were conducted in a single session lasting around forty-five minutes. A male, 15 years-old docile horse (undefined breed $-157 \mathrm{~cm}$ tall) and trained for hippotherapy was used in this experiment. An experienced horse handler led the horse. The main therapist alternated between observing from a few feet away and walking alongside, providing cues. A second therapist walked along on the opposite side as a safety measure. The session was performed in an enclosed indoor riding arena and included the use of hippotherapy complete standard safety procedures. The experimental procedure involved six laps on the riding arena - 60 $m$ length in total, per lap (Figure 1). A comfortable and regular riding speed was used. In total, every lap on the riding arena lasted around 3 minutes. Two laps per condition was used: clockand anticlockwise. Three different conditions were performed in the following order: without vest (NoVest), vest without any tension (Vest) and vest with elastic tension applied on the straps (Vest+Tension). The Vest condition was included to investigate possible differences between sensory stimulation and tensile effects of HippusSuit $®$. HippusSuit $®$ was fully described elsewhere [14] and was positioned to increase trunk extenders tone and to increase trunk extension posture. Lower limbs were not stimulated in this study.

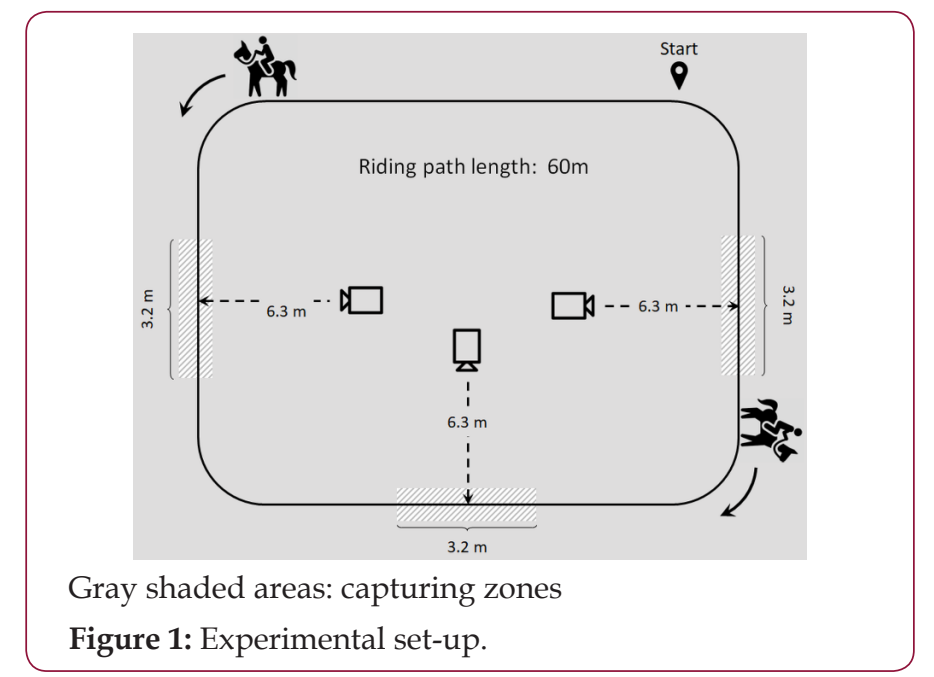

\section{Data Acquisition and Processing}

Four camcorders (Panasonic®, Tokio - Japan - sampling rate of $60 \mathrm{~Hz}$ ), positioned $6.3 \mathrm{~m}$ from the riding path (Figure 1) and $1.2 \mathrm{~m}$ above the ground were used to capture the position of six passive markers attached to the following anatomic landmarks: bilaterally on the acromion, hip joint projection and lateral condyle of femur. Three $3.2 \mathrm{~m}$ capturing zones were used. Markers were digitized automatically on Digital Video for Windows (DVIDEOW) software [16]. As kinematic dependent variable, we assessed the trunk extension angle using MB-Ruler Pro 5.3.2 software. A zerodegree angle meant total trunk flexion. For each capturing zone, the central frame was considered for this analysis. Therefore, for every condition, six measures (three capturing zones, two laps on the riding arena) were considered. 
Muscle activity was recorded at $2000 \mathrm{~Hz}$ with a telemetric system (Delsys $®$ ) with a $10-500 \mathrm{~Hz}$ bandwidth and $1000 \mathrm{x}$ gain. The electromyography (EMG) system was digitally synchronized with the camcorders. Two active electrodes (Delsys ${ }^{\circledR}$ ) were bilaterally positioned on the belly of external oblique muscles according to SENIAM guidelines [17], after proper skin preparation (shaving, abrasion and cleaning). Root mean square (RMS) values were determined for the total time-period the participant was within the same capturing zones used in the kinematic analysis. Since no differences were observed between body-sides, we averaged RMS values for both body sides. Therefore, we determined six RMS values per condition (three capturing zones, two laps). Additionally, three axes accelerometers (embedded in Delsys $\AA$ electrodes) were used to determine the peak acceleration in the three movement planes.
For this analysis, accelerometry data (captured with a sampling frequency of $128 \mathrm{~Hz}$ ) was filtered using a $4^{\text {th }}$ order Buttheworth filter with a cut-off frequency of $10 \mathrm{~Hz}$. All procedures used to determine RMS were repeated for accelerometry data. However, we considered the peak-acceleration within capture zones. Therefore, six peak-acceleration values were determined per condition. All data processing methods were conducted in Matlab (Mathworks@) using specific-designed codes. For statistical analyses the following outcomes were considered: trunk extension angle, EMG RMS and vertical, medio-lateral and antero-posterior peak-accelerations in three planes. Student t-tests for single samples were used, with the NoVest condition as reference value. For this procedure, we considered the average of all values obtained during the NoVest condition as reference. All statistical procedures were conducted in Stastistica 10.0 software (StatSoft $®$ ).
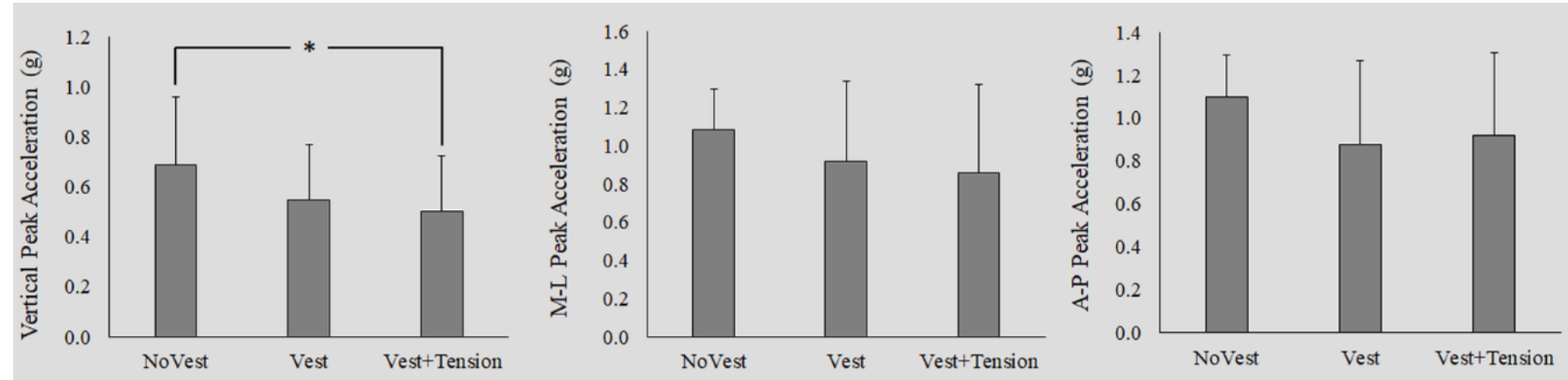

* $\mathrm{p}<0.05$; NoVest: without HippusSuit ${ }^{\circledR}$. Vest: with HippusSuit ${ }^{\circledR}$, but no tension applied; Vest+Tension: HippusSuit ${ }^{\circledR}$ with tension applied.

Figure 2: Peak acceleration in all movement planes, per condition condition.
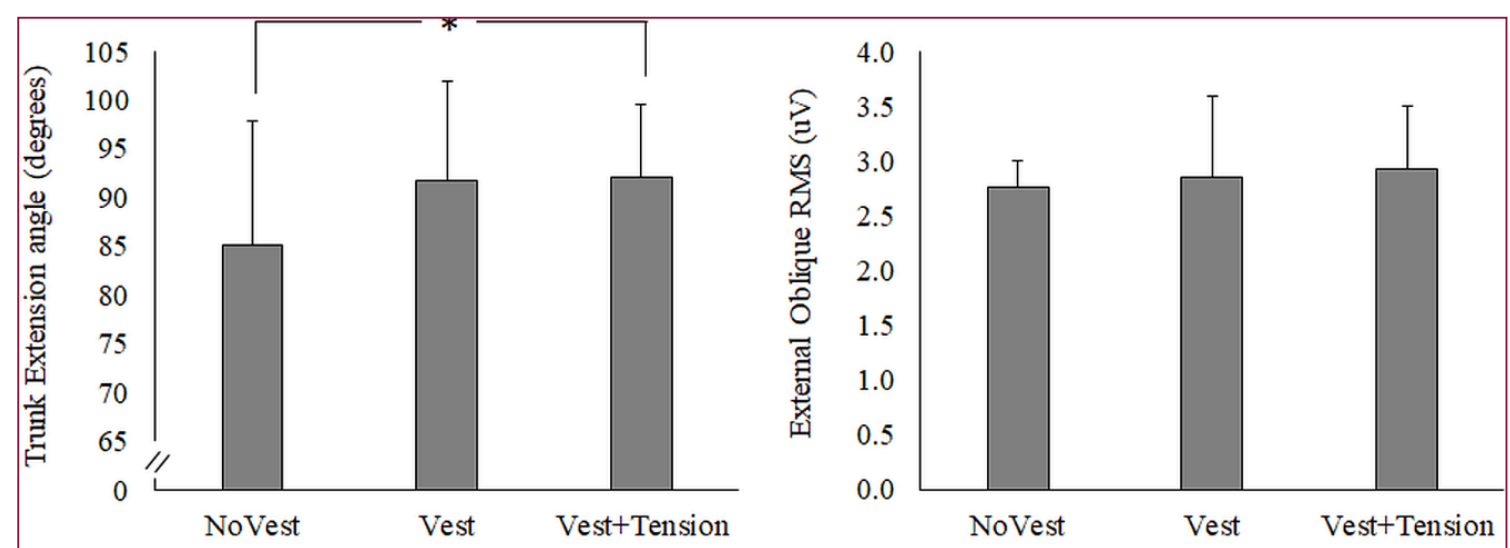

${ }^{*} p<0.05$; RMS: Root Mean Square. NoVest: without HippusSuit ${ }^{\circledR}$. Vest: with HippusSuit ${ }^{\circledR}$, but no tension applied; Vest+Tension: HippusSuit ${ }^{\circledR}$ with tension applied.

Figure 3: Trunk extension angle (left) and External Oblique RMS (left), per condition.

Figure 2 shows the trunk extension angle per condition and the magnitude of muscle activation in all conditions. The results demonstrated a higher trunk extension angle with the use of HippusSuit $₫$ and tension applied on straps in comparison to condition without vest (increase of $8.3 \%$; $=0.007$ ). Although an increase in trunk extension could also be visually observed in the Vest condition (increase of 7.8\%), no statistical significance was found $(\mathrm{p}=0.08)$. For external oblique RMS, no differences were found among conditions ( $\mathrm{p}>0.09)$. These findings indicate an improvement in postural alignment with the use of HippusSuit ${ }^{\circledR}$ without muscle 'accommodation' effect.

Figure 3 demonstrates the peak accelerations of the trunk in the three movement planes. The use of the HippusSuit ${ }^{\circledR}$ with tension reduced the peak acceleration in the vertical direction, $(p=0.025)$. Furthermore, although no statistical significance was 
found ( $p>0.15)$, a reduction in the mid-lateral and anteroposterior acceleration was also observed with the use of HippusSuit@, especially when tension was applied: medio-lateral acceleration: $15.61 \%$ and $21.08 \%$ (Vest and Vest+Tension, respectively); anteroposterior acceleration: $20.28 \%$ and $16.47 \%$. These results indicate an improvement in trunk stability with the use of HippusSuit@.

\section{Discussion}

The aim of this single-case study was to investigate the effects of HippusSuit ${ }^{\circledR}$, on postural alignment, trunk stability and muscle activation during a hippotherapy single-session in a 3-years old girl with a clinical diagnosis of DWS. The results demonstrated an improvement in trunk stability and alignment that cannot be attributed to tactile stimuli, since no effects were observed when HippusSuit $\AA$ was used without elastic tension. Furthermore, since muscle activity remained similar in all conditions, the hypothesis that wearing HippusSuit ${ }^{\circledR}$ could reduce muscle recruitment level due to a disuse effect can be rejected. M.P. attended the hippotherapy center with a clinical diagnostic of DWS, presenting moderate hypotonia, poor trunk stability control and balance impairments. Since hippotherapy has been shown to improve balance [15] and general motor improvements in a variety of neuromuscular disorders [7], including hypotonia [18], it was feasible to believe that M.P. would also benefit from hippotherapy.

However, M.P.'s poor trunk stability control could limit the hippotherapy benefits. In this way, we hypothesized that HippusSuit ${ }^{\circledR}$ would fit perfectly M.P.'s need. Indeed, as result, we observed a more extended trunk position and a reduction in body acceleration, suggesting an improved trunk stability [19]. The literature is very scarce about the effects of any therapeutic/orthotic vests during hippotherapy, what compromises the comparison of our results with others. To our best understanding this is the very first study to assess the effects of a rectification orthosis on trunk stability and postural alignment in a child with DWS. Gomes et al. [14] reported an improvement of body positioning in cerebral palsy individuals with the use of HippusSuit $\circledast$. Furthermore, participants related an improvement of body awareness with the use of HippusSuit ${ }^{\circledR}$ [14]. However, the authors did not provide further information about the instruments used to assess these features and did not report any outcomes values [14]. Therefore, this is the very first study to properly describe the effects of a rectification vest on postural alignment and stability during a single session of hippotherapy.

Interestingly, the muscle recruitment profile observed in our study with the use of HippusSuit $\AA$ is distinct to those reported in cerebral palsy individuals [14]. Gomes et al. [14] reported a reduction in muscle activation levels in erector spinae and rectus abdominis muscles with HippusSuit ${ }^{\circledR}$. The authors related these findings to an improvement in trunk stability provided by the rectification orthosis [14], suggesting a disuse effect. We argue this effect would not be positive to M.P., since she would benefit from hippotherapy by the stimulation of a higher muscle tonus [18]. Fortunately, we did not observe a reduction in external oblique recruitment level with HippusSuit@, suggesting a lack of muscle 'accommodation'. This dissimilarity might be justified by the tensile force applied on lower limbs: Gomes et al. [14], reported an increase in recruitment level of adductor muscles of the hip and we did not apply any tensile force on lower limbs straps, since we were interested solely on trunk effects. Gomes et al. [14] suggested that an increase in the adductor muscles activity might had contributed to the 'accommodation' effect observed on trunk muscles. Therefore, since we did not include tensile force on lower limbs, this might justify the discrepancy of results between both studies. However further investigations should be conducted to clarify this issue.

Some limitations should be noted as:

a) We did not assess the recruitment level of other muscles rather than external oblique. Although we had intended to assess these muscles the vest itself limited the signal acquisition. Further methods might be implemented in future studies allowing the assessment of other muscles.

b) This is a single-case study and caution is advised before final recommendations are made.

c) Although head behavior (posture and stability) was closely controlled by therapists, we did not assess these features. This should be considered in futures studies.

We conclude that HippusSuit ${ }^{\circledR}$ is effective to improve trunk alignment and stability in a child with DWS during a hippotherapy session. These effects were not accompanied by an 'accommodation' / disuse effect of trunk muscles. Finally, it is important to note that all effects observed in our study cannot be attributed to tactile stimulation, since no effects were observed when the HippusSuit ${ }^{\circledR}$ vest was applied without any tensile force. HippusSuit ${ }^{\circledR}$ might be an interesting instrument to assist practitioners with hypotony during hippotherapy.

\section{Acknowledgement}

This study was financed in part by the Coordenação de Aperfeiçoamento de Pessoal de Nível Superior - Brasil (CAPES) Finance Code 001. Additionally, CAPES provided final support as scholarship (Grant \#: N/A).

\section{References}

1. Dandy WE (1921) The diagnosis and treatment of hydrocephalus due to occlusion of the foramina of Magendie and Luschka. Surg Gynec Obst 32: $112-124$.

2. Zamora EA, T Ahmad (2019) Dandy Walker Malformation, in Stat Pearls. Treasure Island (FL).

3. Ameli NO (1966) Arrest of development and dandy-walker malformation. Brain 89(3): 549-554.

4. Figueroa Ángel V, Reyes Moreno I, García López R, Rodríguez Aceves C, Martínez Muñiz I (2013) Síndrome de Dandy-Walker. Arch Neurocien (Mex) 18(2): 92-98.

5. Rodriguez Virgili J, AA Cabal Garcia (2010) Dandy Walker syndrome. Aten Primaria 42(1): 50-51.

6. Mohanty A, Biswas A, Satish S, Praharaj SS, Sastry KV (2006) Treatment options for Dandy-Walker malformation. J Neurosurg 105(5 Suppl): 348-356. 
7. Koca TT, H Ataseven (2015) What is hippotherapy? The indications and effectiveness of hippotherapy. North Clin Istanb 2(3): 247-252.

8. Martin-Valero R, J Vega Ballon, V Perez Cabezas (2018) Benefits of hippotherapy in children with cerebral palsy: A narrative review. Eur J Paediatr Neurol 22(6): 1150-1160.

9. Lucena-Anton D, I Rosety-Rodriguez, JA Moral-Munoz (2018) Effects of a hippotherapy intervention on muscle spasticity in children with cerebral palsy: A randomized controlled trial. Complement Ther Clin Pract 31: 188-192.

10. Ajzenman HF, JW Standeven, TL Shurtleff (2013) Effect of hippotherapy on motor control, adaptive behaviors, and participation in children with autism spectrum disorder: a pilot study. Am J Occup Ther 67(6): 653663.

11. Munoz-Lasa S, Ferriero G, Valero R, Gomez-Muñiz F, Rabini A, et al (2011) Effect of therapeutic horseback riding on balance and gait of people with multiple sclerosis. G Ital Med Lav Ergon 33(4): 462-467.

12. Guerino MR, AF Briel, M Araujo (2015) Hippotherapy as a treatment for socialization after sexual abuse and emotional stress. J Phys Ther Sci 27(3): 959-962.

13. Gomes TT, RM Hassunuma, LM Silva (2014) Equine therapy as a therapeutic resource in myelomeningocele: a case study. Rev Neurocienc 22(3): 458-463.

\section{ISSN: 2574-1241}

DOI: 10.26717/BJSTR.2019.17.002953

Marcelo Pinto Pereira. Biomed J Sci \& Tech Res

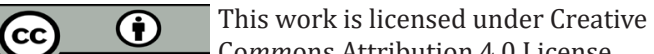

Submission Link: https://biomedres.us/submit-manuscript.php
14. Gomes AAA, CF Lima, K Hollatz (2017) Development of a postural rectification orthosis as a recruiter in the practice of equine therapy, in Equine therapy: theory and practice in Brazil, DFG Soares, et al. (Eds.), FUNEC Editora: Caratinga, pp. 393-404.

15. Erdman EA, SR Pierce (2016) Use of Hippotherapy With a Boy After Traumatic Brain Injury: A Case Study. Pediatr Phys Ther 28(1): 109-116.

16. Figueroa PJ, NJ Leite, BRM (2003) A flexible software for tracking of markers used in human motion analysis. Comput Methods Programs Biomed 72(2): 155-165.

17. Merletti R, H Hermens (2000) Introduction to the special issue on the SENIAM European Concerted Action. J Electromyogr Kinesiol 10(5): 283-286.

18. Hsieh Y, Shu-Ya Zhan, Shyi-Kuen Wu, Chen-Chia Yang, Yu-Chun Lee, et al. (2008) Hippotherapy on Gross Motor Function in a Child with Hypotonic Quadriplegic Cerebral Palsy: A 1-year Follow-up Tw J Phys Med Rehabil 36(3): 177-185.

19. Martinez-Mendez R, M Sekine, T Tamura (2012) Postural sway parameters using a triaxial accelerometer: comparing elderly and young healthy adults. Comput Methods Biomech Biomed Engin 15(9): 899910.

$\begin{array}{ll}\text { BIOMEDICAL } & \text { Assets of Publishing with us } \\ \text { RESEARCHES } & \text { - Global archiving of articles } \\ & \text { - Immediate, unrestricted online access } \\ & \text { - Rigorous Peer Review Process } \\ \end{array}$

